\title{
Remoción de Calcio y Magnesio en Agua de Mar para Mejorar la Concentración de Sólidos en la Descarga de Espesadores
}

\author{
Ricardo I. Jeldres ${ }^{(1)}$, Eder C. Piceros ${ }^{(2)}$, Julio A. Valenzuela ${ }^{(3)}$, y Pedro A. Robles ${ }^{(4)}$ \\ (1) Departamento de Ingeniería Química y Procesos de Minerales, Facultad de Ingeniería, Universidad de Antofagasta, PO \\ Box 170, Antofagasta, Chile. (e-mail: ricardo.jeldres@uantof.cl) \\ (2) Facultad de Ingeniería y Arquitectura, Universidad Arturo Prat, PO BOX 121, Iquique, Chile. (e-mail: edpicero@unap.cl) \\ (3) Departamento de Ingeniería Química, Universidad Católica del Norte, Antofagasta, Chile. (e-mail: jvalenzuela01@ucn.cl) \\ (4) Escuela de Ingeniería Química, Pontificia Universidad Católica de Valparaíso, Valparaíso, Chile. \\ (e-mail: pedro.robles@pucv.cl) \\ * Autor a quien debe ser dirigida la correspondencia
}

Recibido Feb. 7, 2019; Aceptado Abr. 12, 2019; Versión final May. 15, 2019, Publicado Oct. 2019

\begin{abstract}
Resumen
Con el objetivo de aumentar la concentración de sólidos de sedimentos de un relave minero, se utilizó agua de mar con cantidades reducidas de calcio y magnesio. Los experimentos se efectuaron considerando un relave sintético, compuesto por cuarzo y caolinita, y utilizando un floculante aniónico de alta masa molecular. El agua de mar generó un comportamiento eficiente al trabajar a $\mathrm{pH}<9$. Sin embargo, a mayor alcalinidad la concentración de sólidos del sedimento se redujo sustancialmente. Examinando los principales iones por separado se encontró que el magnesio, y en menor medida el calcio, son los principales responsables de reducir la eficiencia del proceso, producto de la formación de complejos $\mathrm{MgOH}^{+} / \mathrm{CaOH}^{+}$y precipitados de $\mathrm{Mg}(\mathrm{OH})_{2}$. Por este motivo, al utilizar el agua de mar con concentraciones reducidas de calcio y magnesio se logró aumentar considerablemente la concentración de sólidos, ofreciendo una alternativa eficaz para operar a condiciones altamente alcalinas.
\end{abstract}

Palabras clave: agua de mar; arcillas; calcio y magnesio; espesadores; relaves

\section{Removal of Calcium and Magnesium from Seawater to Increase the Solid Concentration in Thickener Underflow}

\begin{abstract}
Seawater with reduced amounts of calcium and magnesium was used for increasing the concentration of sediment solids from a mine tailing. The assays were carried out considering a synthetic tailing, composed of quartz and kaolinite, and utilising an anionic flocculant of high molar weight. Seawater produced an efficient behaviour when working at $\mathrm{pH}<9$. However, at higher alkalinity the solid concentration of the sediment was substantially reduced. Isolating the effect of the main seawater ions it was found that magnesium, and to lesser extent calcium, are responsible for reducing the flocculation efficiency, caused by the formation of $\mathrm{MgOH}^{+} / \mathrm{CaOH}^{+}$complexes and $\mathrm{Mg}(\mathrm{OH})_{2}$ precipitates. For this reason, when using seawater with a reduced amount of calcium and magnesium, it was possible to considerably increase the solids content, offering an effective alternative to operate at highly alkaline conditions.
\end{abstract}

Keywords: seawater; clays; calcium and magnesium; thickeners; tailings 


\section{INTRODUCCIÓN}

Si bien la implementación directa de agua de mar en la industria minera involucra numerosos desafíos operacionales, la necesidad de enfrentar los problemas de escasez de agua genera que en muchas zonas sea la única opción viable para conservar la sustentabilidad de este sector productivo (Cisternas y Gálvez, 2018; Hernández et al., 2012; Ordóñez et al., 2013). Las distancias y altitudes geográficas a las que se debe transportar el agua desde la costa hasta donde se ubican los yacimientos suelen ser considerablemente altas, involucrando costos de bombeo significativos. Por lo tanto, para equilibrar la demanda energética es imperante maximizar la eficiencia de las etapas de recuperación del recurso hídrico, siendo el espesamiento la operación que adquiere mayor relevancia. Esta operación se fundamenta en formar agregados masivos de partículas que sedimenten por efectos gravitacionales, y de esta manera generar un underflow espesado y un overflow de agua clarificada. La formación de agregados se logra principalmente mediante floculantes, que corresponden a polímeros solubles de alto peso molecular, los cuales se pueden adherir a una gran cantidad de minerales.

La información disponible acerca del impacto que provoca el agua de mar en etapas de espesamiento es escasa. Sin embargo, se ha reportado que la salinidad genera efectos muy diversos y complejos durante la floculación de minerales (Antunes et al., 2008; Colic et al., 1997; Jeldres et al., 2014; Pan y Yoon, 2018). Por ejemplo, se han mostrado casos en que la presencia de electrolitos mejora la agregación de partículas por la reducción de repulsiones electrostáticas, mientras que en otras circunstancias una elevada salinidad perjudicaría la formación de flóculos (Ji et al., 2013; Lee et al., 2012; Liu et al., 2018). Tales discrepancias fueron analizadas por Jeldres et al. (2017b). Los autores explicaron que la salinidad tiene un efecto no monótono sobre la floculación de partículas dado que aparecen diferentes fenómenos competitivos que pueden dañar o mejorar el crecimiento de agregados de acuerdo a las circunstancias. Por una parte, un medio salino favorece la adsorción de la poliacrilamida a la superficie del mineral debido a la compresión de la atmósfera iónica que rodea a los coloides y a los enlaces extras que proveen los iones, que por lo general pueden formar un puente catiónico entre las poliacrilamidas aniónicas y los minerales. Por otro lado, el floculante reduce su tamaño en solución debido al apantallamiento de la carga eléctrica de sus grupos funcionales, limitando el tamaño máximo que los flóculos podrían alcanzar. Estos fenómenos fueron validados recientemente por Quezada et al. (2018), quienes utilizaron simulaciones de dinámica molecular para analizar la interacción de una poliacrilamida aniónica con una superficie de cuarzo en soluciones salinas.

Sin embargo, la fuerza iónica no es el único aspecto que se debe considerar respecto al agua de mar, ya que a condiciones altamente alcalinas los iones de calcio y magnesio se hidrolizan en la forma de $\mathrm{CaOH}^{+} / \mathrm{MgOH}^{+}$ y surgen complejos que precipitan como $\mathrm{Ca}(\mathrm{OH})_{2} / \mathrm{Mg}(\mathrm{OH})_{2}$. Estas especies, cuya concentración depende del $\mathrm{pH}$ y la concentración inicial de cada elemento, han mostrado ser muy perjudiciales en operaciones de flotación de sulfuros, ya que su capacidad para adherirse a la superficie de algunos minerales conduce a que estos pierdan hidrofobicidad y consecuentemente disminuyan su flotabilidad (Castro, 2012; Jeldres et al., 2017a, 2016; Li et al., 2018; Ruan et al., 2018). Menor es la información disponible acerca del impacto que genera la formación de estos complejos en operaciones de espesamiento. Sin embargo, se conoce que iones polivalentes tienen una influencia significativa en los mecanismos de floculación. Por ejemplo, Peng y Di (1994) mostraron que los cationes de $\mathrm{Ca}^{2+} \mathrm{y} \mathrm{CaOH}^{+}$pueden ser adsorbidos por el grupo carboxil de las PAMA, formando complejos como $-(\mathrm{COO})_{2} \mathrm{Ca}$ o $\mathrm{COOCa}(\mathrm{OH})$, dependiendo del tipo de catión adsorbido. Esto tiene como consecuencia una disminución de los grupos funcionales disponibles de la PAM y por tanto un detrimento en su capacidad para generar puentes poliméricos. Así mismo, los complejos de calcio y magnesio podrían cubrir parte de la superficie activa de los minerales.

Mpofu et al. (2003) estudiaron el efecto de iones de calcio sobre el potencial zeta de caolinita, demostrando

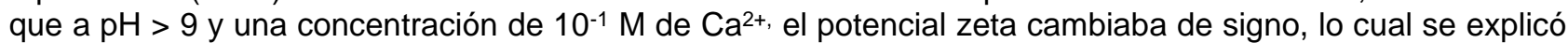
en términos de la adsorción de especies de calcio hidrolizadas (e.g. $\mathrm{Ca}(\mathrm{OH})^{+}$) o incluso precipitación de $\mathrm{CaOH}^{2}$ en la superficie del mineral. Esto también fue propuesto anteriormente por Atesok et al. (1988) para partículas de la misma naturaleza. Zbik et al., (2008) utilizaron microfotografías cri-SEM para estudiar el impacto del floculante e iones de calcio sobre la conformación de agregados de caolinita. Los autores mostraron que la estructura de los agregados cuando se forman en ambiente libre de floculante es en gran medida preservada al adicionar un polielectrolito. Sin embargo, la presencia de iones de calcio generó agregados con estructuras significativamente diferentes. En este sentido, Jeldres et al. (2017b) reportaron que la presencia de electrolitos genera flóculos de caolinita más compactados en comparación a flóculos generados en agua destilada. Avadiar et al. (2015) analizaron la influencia de la hidrolización de cationes de calcio y magnesio sobre el potencial zeta de cuarzo, alúmina, y caolín. Los autores encontraron que los complejos son capaces de adherirse a la superficie de los tres minerales, ocasionando un impacto significativo en su carga superficial ya que elevan el valor del potencial zeta, logrando incluso valores positivos al estar por sobre el punto isoeléctrico de los minerales. 
Si bien se han realizado numerosos esfuerzos por explicar los mecanismos con que interactúan diferentes tipos de iones en la floculación de los principales minerales que componen los relaves, no hay un estudio sistemático que aborde el efecto del agua de mar sobre la concentración de sólidos de la corriente de descarga de los espesadores (underflow); siendo esta una propiedad fundamental para definir la cantidad máxima de agua que puede ser recuperada en esta operación. En este estudio, se analiza el impacto del agua de mar a diferentes condiciones de pH sobre la concentración de sólidos de los sedimentos de relaves, floculados con polímero aniónico de alto peso molecular. Posteriormente se evaluó el uso de agua de mar tratada, reduciendo su contenido de calcio y magnesio mediante una atmósfera rica en $\mathrm{CO}_{2}$ y utilizando $\mathrm{NaOH}$ como agente alcalinizante.

\section{MATERIALES Y MÉTODOS}

Se utilizó caolinita de alta pureza (KGA-1b, Acros Organics S.A.) con densidad de $2.6 \mathrm{~g} / \mathrm{cm}^{3}$. Su diámetro promedio fue $12 \mu \mathrm{m}$. El cuarzo fue adquirido en 'donde Capó' y clasificado a modo de que el $100 \%$ de las partículas esté bajo malla \#200. Se utilizó un floculante comercial, consistente en una poliacrilamida de peso molecular $18 \times 10^{6} \mathrm{~g} / \mathrm{mol}$. Para lograr una solución polimérica homogénea, el floculante se agregó paulatinamente al agua hasta obtener una concentración de $1 \mathrm{~g} / \mathrm{L}$, y sometido a agitación magnética por 24 hrs. La muestra fue almacenada en oscuridad y a $8^{\circ} \mathrm{C}$ para evitar su degradación. Una vez al día se tomó una alícuota de la solución madre de floculante y se diluyó a $0.1 \mathrm{~g} / \mathrm{L}$ para aplicarla en los experimentos a temperatura ambiente. El agua de mar empleada para desarrollar los ensayos experimentales se obtuvo de la bahía San José en Antofagasta (Chile). Su composición química se muestra en la Tabla 1. El resto de los reactivos empleados (sales) fueron de grado analítico.

Tabla 1: Composición química de agua de mar de Bahía San José.

\begin{tabular}{|c|c|c|c|}
\hline lones & Valor $(\mathrm{mg} / \mathrm{L})$ & lones & Valor $(\mathrm{mg} / \mathrm{L})$ \\
\hline Magnesio & 1413 & Bicarbonato & 137 \\
\hline Calcio & 391 & Fosfato & $<0.20$ \\
\hline Sodio & 11100 & Sulfato & 2795 \\
\hline Potasio & 389 & Nitrato & 1.90 \\
\hline Cloruro & 19888 & Fluoruro & 0.888 \\
\hline
\end{tabular}

\section{Potencial zeta}

Se preparó una solución de mineral (cuarzo o caolín) $0.1 \%$ p/p y el pH se ajustó mediante $\mathrm{HCl}$ o $\mathrm{NaOH}$ según correspondiera. Se adicionó $0.001 \mathrm{M}$ de NaCl para minimizar la influencia de los iones $\mathrm{Na}^{+}$y $\mathrm{Cl}^{-}$cuando se emplean los agentes modificadores de pH. Los ensayos se realizaron en un equipo Zetameter 4.0.

\section{Tratamiento de agua de mar: remoción de calcio y magnesio utilizando $\mathrm{CO}_{2}$ y $\mathrm{NaOH}$}

El tratamiento de agua de mar permite reducir la cantidad de iones de calcio y magnesio, adicionando $\mathrm{NaOH}$ en una atmósfera rica en $\mathrm{CO}_{2}(\mathrm{~g})$. Los ensayos experimentales se desarrollaron introduciendo $2 \mathrm{~L}$ de agua de mar en un matraz a temperatura ambiente y agitación continua. Primero, se adicionó la solución de $\mathrm{NaOH}$ utilizando un titulador potenciométrico (Shott Tritoline 700). Luego, se inyectó $\mathrm{CO}_{2}(\mathrm{~g})$ durante un periodo de tiempo específico a través de un tubo de inmersión utilizando un rotámetro automático (Alicat Scientific, 1-100 $\mathrm{mL} / \mathrm{min}$ ) junto con $\mathrm{NaOH}$ para mantener un $\mathrm{pH}$ estable de la solución $(\mathrm{pH} 10.5)$. Finalmente el agua fue filtrada con un sistema de filtración al vacío con membrana de $0.22 \mu \mathrm{m}$ (Merk Millipore) y posteriormente caracterizada mediante Espectrometría de Absorción Atómica para determinar la concentración remanente de calcio y magnesio.

\section{Ensayos de sedimentación}

Los experimentos de floculación se efectuaron en un agitador tipo turbina PTFE $30 \mathrm{~mm}$, colocado en posición axial, en recipiente de $100 \mathrm{~mm}$ de diámetro y $1 \mathrm{~L}$ de capacidad. Se adicionó un volumen de solución de 200 $\mathrm{mL}$, seguido por $32.5 \mathrm{~g}$ de relave sintético, compuesto por $30 \mathrm{~g}$ cuarzo y $2.5 \mathrm{~g}$ de arcilla. La pulpa fue preacondicionada mezclando a $450 \mathrm{rpm}$ por $60 \mathrm{~min}$. Tras ese periodo, se redujo la tasa de agitación a $125 \mathrm{rpm}$ y se adicionó una cantidad de floculante de acuerdo a la dosis respectiva. Adicionalmente se agregó un volumen de agua residual para lograr una pulpa de masa total de $250 \mathrm{~g}$, con una concentración de sólidos de $13 \%$ p/p en todos los casos. La suspensión se mezcló con el floculante durante $60 \mathrm{~s}$ y luego se vació 
suavemente en la columna de sedimentación de $35 \mathrm{~mm}$ de diámetro interno. Luego, el cilindro se invirtió 2 veces de forma manual para asegurar la homogeneidad de la suspensión floculada. La concentración de sólidos del sedimento se calculó mediante un balance de masa, utilizando la altura final del sedimento cuando transcurrió una hora desde que comenzó la sedimentación. Todas las experiencias fueron triplicadas y en el presente estudio se muestra el valor promedio de las mediciones.

\section{RESULTADOS}

A continuación se presentan los resultados obtenidos en este trabajo, clasificados en tres subsecciones: la primera expresa el tipo de carga eléctrica de las partículas que componen el relave sintético; la segunda muestra la concentración de sólidos obtenida en los sedimentos que se constituyen en diferentes tipos de agua y condiciones de $\mathrm{pH}$. Se observa el efecto de incluir agua de mar tratada con remoción de calcio y magnesio; en la tercera subsección se hace una descripción sobre las implicancias operacionales que generan los resultados reportados en el presente artículo.

\section{Potencial zeta}

Las mediciones de potencial zeta de cuarzo y caolinita se realizaron sobre un rango de pH entre 2 y 11.5 , y los resultados se presentan en la figura 1. Los resultados muestran que los dos minerales que componen el relave sintético tienen un potencial zeta negativo en la mayoría de los pHs analizados, lo cual es consistente con la naturaleza fisicoquímica de la superficie de ambos minerales. En el caso de la caolinita, el potencial zeta negativo refleja la magnitud global de la superficie, indicando el predominio de las caras (compuestas por grupos de silanol) por sobre los bordes (principalmente aluminol).

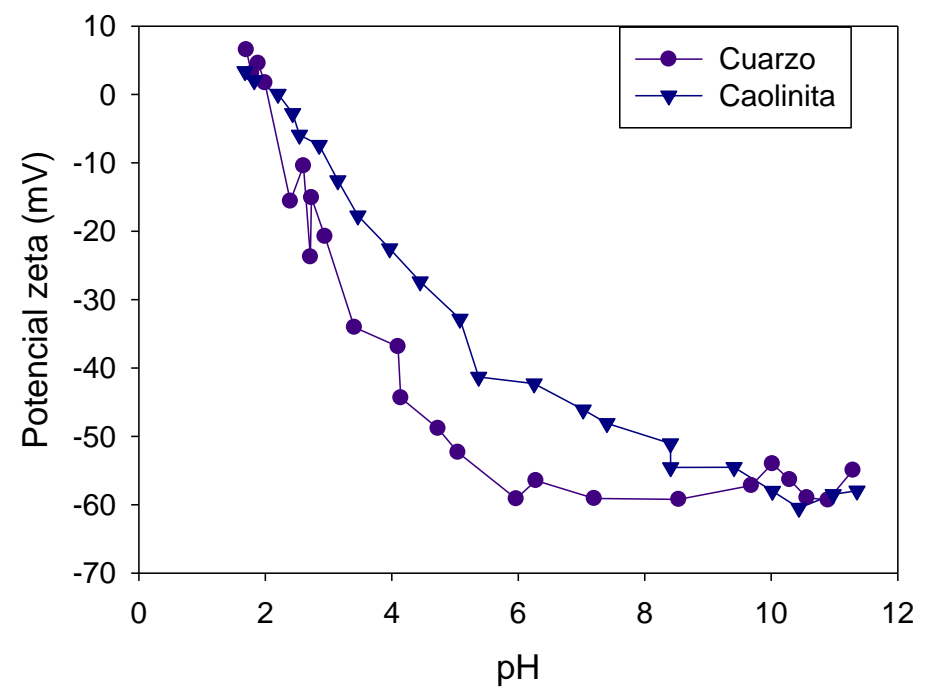

Fig. 1: Efecto del pH sobre el potencial zeta de partículas de cuarzo y caolinita en agua destilada con presencia de $\mathrm{NaCl} 0.001 \mathrm{M}$.

\section{Concentración de sólidos en sedimentos}

En la figura 2 se analiza el efecto del pH sobre el porcentaje de sólidos del sedimento del relave floculado en distintos tipos de agua. Se observa que a un pH menor a 9, el contenido de sólidos en el sedimento alcanza un valor cercano al $60 \% \mathrm{p} / \mathrm{p}$, para todos los tipos de aguas salinas. Sin embargo, a condiciones altamente alcalinas, en este caso $\mathrm{pH}>9$, la pulpa preparada en agua de mar sufre un deterioro drástico, reduciendo el porcentaje de sólidos a valores cercanos al 30\% p/p. Al analizar los principales cationes que componen el agua de mar por separado $\left(\mathrm{Na}^{+}, \mathrm{Mg}^{+2}, \mathrm{Ca}^{+2}\right)$, se encuentra que la salinidad en sí no es perjudicial para el proceso, de hecho, un medio salino no sería un problema mayor para la floculación. Si bien la salinidad perjudica la formación de flóculos debido al enrollamiento que sufre el floculante producto del apantallamiento electrostático de sus sitios activos, también existe un efecto positivo para el proceso ya que los cationes presentes en el medio pueden formar un enlace iónico extra, que une a las cargas aniónicas de las partículas de cuarzo y caolinita con la carga aniónica del floculante. En este sentido, la salmuera de $\mathrm{NaCl}$ no generó mayores dificultades, sin embargo, se observa que el magnesio es el principal responsable de afectar el proceso y reducir la cantidad de sólidos contenida en el sedimento. Es llamativo que la respuesta de la pulpa en una salmuera de $\mathrm{MgCl}_{2} \mathrm{presente}$ un comportamiento muy simular al obtenido en agua de mar. Interesantemente, los resultados coinciden con la formación de complejos de $\mathrm{MgOH}^{+} / \mathrm{CaOH}^{+}$y con los fenómenos de precipitación de los cationes de magnesio a través del compuesto $\mathrm{Mg}(\mathrm{OH})_{2}$ (ver especiación de sales divalentes en Suyantara et al., 2018). 


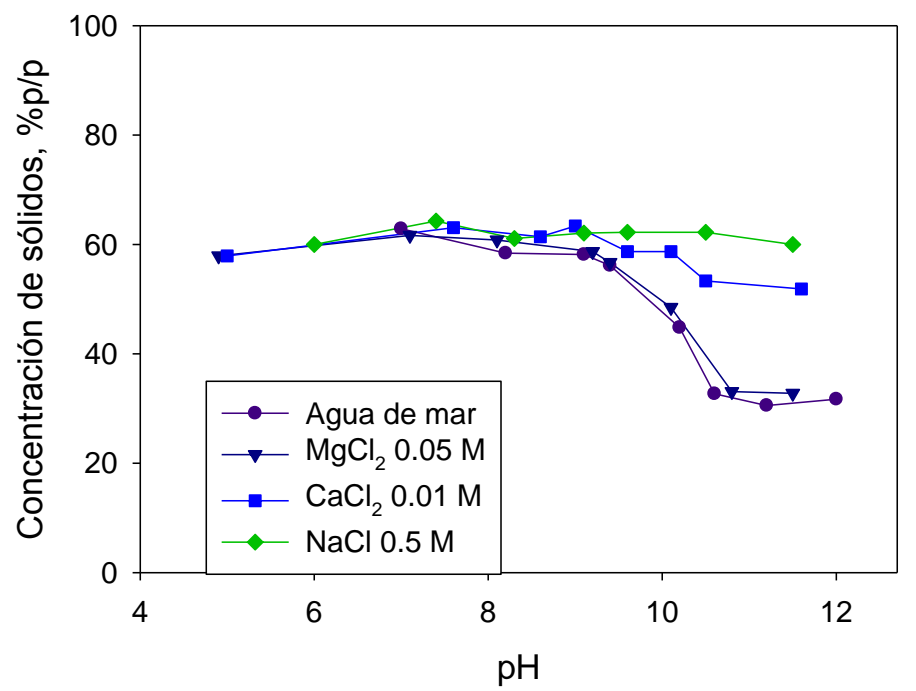

Fig. 2: Concentración de sólidos del sedimento de pulpas floculadas en diferentes medios acuosos como función del pH. Concentración de sólidos en pulpa: 13 \% p/p; dosis de floculante: $20 \mathrm{~g} / \mathrm{ton}$.

En la figura 3 se muestra el efecto de la dosis de floculante sobre el porcentaje de sólidos de los sedimentos cuando las pulpas fueron preparadas en agua de mar. Se compararon los resultados analizando dos condiciones de $\mathrm{pH}: \mathrm{pH}$ natural $(\mathrm{pH}$ 7.8) y $\mathrm{pH}$ 10.5. Las muestras floculadas a $\mathrm{pH}$ natural generan un alto contenido de sólidos, cercano a un $60 \% \mathrm{p} / \mathrm{p}$ en todos los casos, con la salvedad de que un aumento en la dosificación de polímero genera una mayor altura del sedimento final, como lo indica la disminución del porcentaje de sólidos. Este hecho concuerda con el trabajo reportado por Maclver y Pawlik (2017), quienes indicaron que el estado de agregación es un contribuidor clave para determinar la densidad del sedimento. Por lo tanto, un incremento en el grado de floculación de una suspensión debido a una mayor dosificación de polímero producirá un mayor volumen de sedimento, con menor densidad después de la sedimentación. Este aspecto puede ser relevante para diseñar sistemas de depósitos de relaves, puesto que flóculos grandes y de baja densidad (alto contenido de agua) pueden presentar propiedades compresivas que permitan continuar la recuperación de agua directamente desde el depósito. Sin embargo, para establecer los criterios del diseño se requiere conocer los eventuales rangos de 'pendiente de playa' que presentaría el relave espesado, así como información sobre la reología del sistema, que puede ser extraída mediante ensayos de laboratorio (De Araujo et al., 2006).

Cuando la pulpa se llevó a pH 10.5, hubo una gran reducción en el contenido de sólidos del sedimento, siendo aproximadamente un tercio más bajo en la mayoría de los casos, y conservando la tendencia respecto a la dosificación del polímero. Este es un fenómeno que claramente perjudica la eficiencia del proceso, ya que trabajar en estas condiciones de pH significaría que una considerable cantidad de agua se perdería en la corriente de descarga del espesador. En la figura 4 se analiza el porcentaje de sólidos del sedimento resultante en diferentes medios salinos a $\mathrm{pH}$ 10.5. Independiente de la dosificación de polímero, se comprueba que el magnesio, y en menor medida el calcio, son los principales responsables de reducir la eficiencia del proceso, por tanto se optó por utilizar un agua de mar tratada, reduciendo su dureza mediante una atmósfera rica en $\mathrm{CO}_{2}(\mathrm{~g})$ y $\mathrm{NaOH}$ como reactivo alcalinizante. Un análisis químico del agua de mar, antes y después del tratamiento, reveló que la presencia de calcio se redujo de 400 ppm a 134 ppm y el magnesio de 1250 ppm a 75 ppm.

Cabe señalar que existen diferentes técnicas para promover la remoción de calcio y magnesio del agua de mar (Arias et al., 2018; Castro, 2010; Jeldres et al., 2017a). Por ejemplo, Jeldres et al. (2017a) utilizaron una mezcla de dos reactivos alcalinizantes (cal y carbonato de sodio) para promover la formación de complejos de calcio y magnesio que precipiten a pH sobre 10.5. Los autores lograron mejorar considerablemente la flotabilidad de minerales de Cu-Mo, sin embargo, el efecto buffer del agua de mar implicó una adición muy elevada de reactivos para alcanzar los valores de $\mathrm{pH}$ requeridos en la operación. En tal estudio se utilizó una combinación de los reactivos (50-50\%), dando una concentración global de $0.1 \mathrm{M}$, que puede ser excesiva para aplicaciones industriales. En este sentido, la inyección continua de $\mathrm{CO}_{2}(\mathrm{~g})$ en agua de mar promueve la formación de especies como carbonato de calcio $\left(\mathrm{CaCO}_{3}\right)$ y carbonato de magnesio $\left(\mathrm{MgCO}_{3}\right)$ (Al-Anezi et al., 2006), sin necesidad de incluir cantidades abundantes de modificadores de $\mathrm{pH}$, conduciendo a una remoción significativa de los iones de calcio y magnesio. 
Los resultados presentados en la figura 4 muestran que al utilizar un agua de mar tratada se logró aumentar significativamente la concentración de sólidos del sedimento, alcanzando valores similares a los obtenidos en agua de mar a pH natural. Por ejemplo, para una dosificación de $13 \mathrm{~g} / \mathrm{ton}$, el porcentaje de sólidos del sedimento en agua de mar a $\mathrm{pH} 10.5$ fue de $42 \% \mathrm{p} / \mathrm{p}$, pero al utilizar agua de mar pretratada al mismo $\mathrm{pH}$ se obtuvo un $62 \% \mathrm{p} / \mathrm{p}$. Interesantemente, Peng y Di (1994) mostraron que los complejos hidroxo de iones multivalentes pueden interaccionar con los grupos funcionales de las poliacrilamidas, inhibiendo la formación de puentes de hidrógeno entre el floculante y los minerales, reduciendo la eficiencia del polielectrolito. A su vez, estudios recientes han mostrado que los precipitados de calcio y magnesio pueden adherirse a la superficie de los minerales (ver Uribe et al., 2017), cubriendo porciones que potencialmente estarían disponibles para interactuar con floculantes.

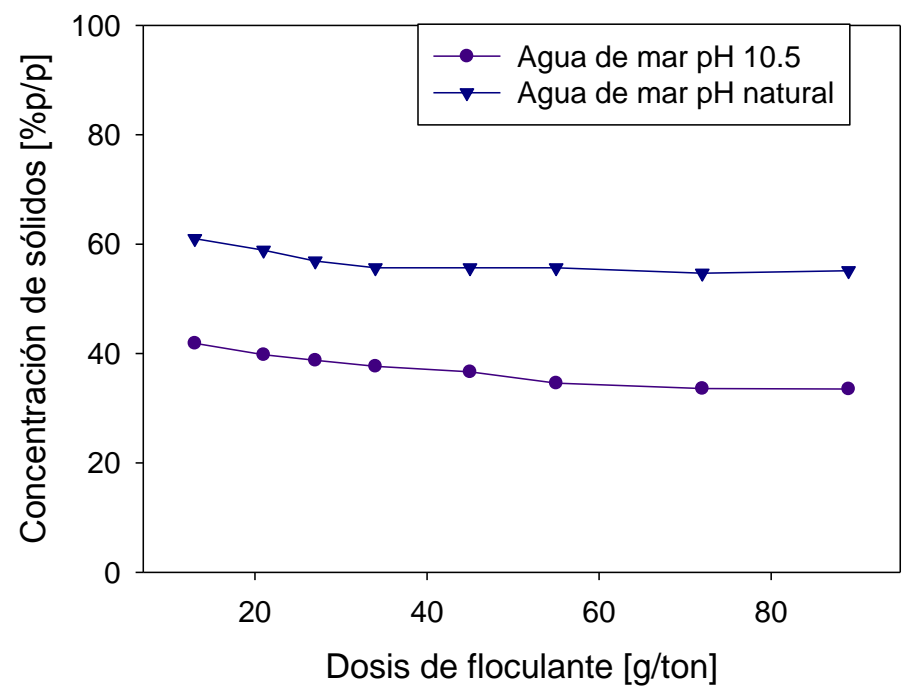

Fig. 3: Efecto de la dosis de floculante y pH en la concentración de sólidos de sedimento de relave en agua de mar. Concentración de sólidos en pulpa: 13\% p/p.

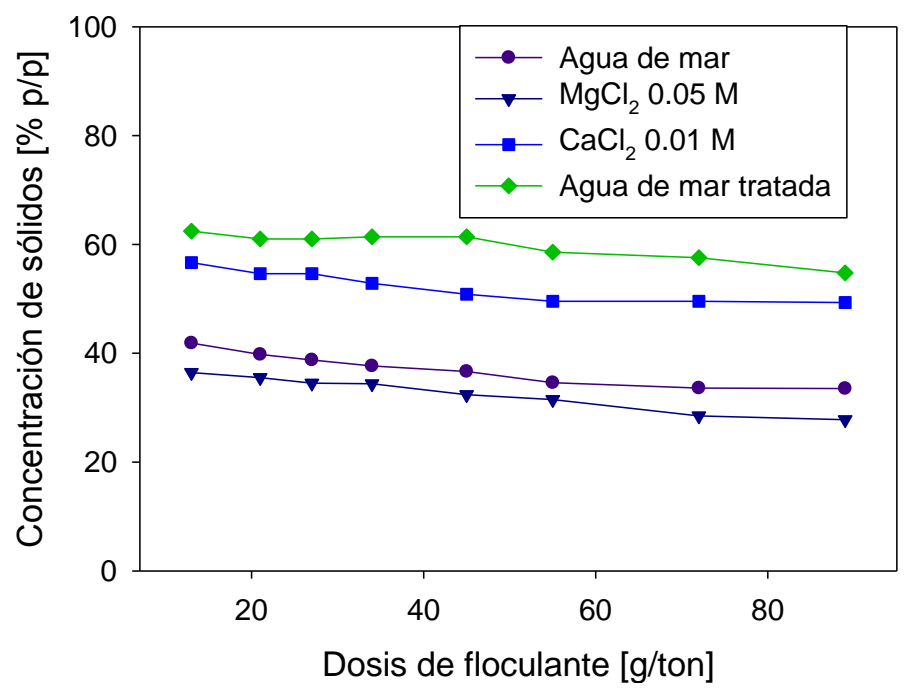

Fig. 4: Efecto de la dosis de floculante en concentración de sólidos de sedimento de relaves en diferentes medios acuosos a pH 10.5. Concentración de sólidos en pulpa: 13\% p/p.

\section{Implicancias operacionales}

La utilización de agua de mar tratada, reduciendo su contenido de calcio y magnesio, ha mostrado mejoras significativas en la flotación de minerales de Cu-Mo en condiciones altamente alcalinas. Sin embargo, hasta la fecha no se han reportado las implicancias que esto genera en operaciones de espesamiento, específicamente en la densidad de la corriente de descarga de los espesadores (underflow). Los resultados obtenidos en este trabajo muestran que la presencia de cationes divalentes afecta el rendimiento de los floculantes desde el pH en que comienza la formación de precipitados. En literatura se ha sugerido que estos complejos hidroxo interaccionan con los grupos funcionales de las poliacrilamidas, disminuyendo la cantidad de enlaces entre el reactivo y el mineral. Con esto, la implementación de una nueva estrategia para remover 
las sales divalentes puede extender los beneficios en las etapas de concentración ya que permitiría generar corrientes de underflow con mayor concentración de sólidos, y por tanto ofrece mayores posibilidades de maximizar la recuperación del recurso hídrico. Una característica interesante de destacar es que el impacto que genera el cloruro de calcio es bastante menor en comparación al cloruro de magnesio. El principal motivo es que las reacciones de precipitación de calcio ocurren a $\mathrm{pH}$ sobre 11, por lo tanto si se reemplaza el hidróxido de sodio por un agente alcalinizante de menor costo como cal, es posible que los beneficios para la operación continúen siendo sustentables.

Este estudio ofrece una temática interesante que debe ser profundizada en futuras investigaciones, principalmente ampliando las condiciones mineralógicas del relave y evaluando las respuestas en las propiedades reológicas del sistema, lo que incide directamente en los costos por transporte y deposición de los relaves espesos (De Kretser et al, 1997).

\section{CONCLUSIONES}

Se analizó el efecto de utilizar agua de mar a condiciones alcalinas sobre el porcentaje de sólidos de sedimentos de relave sintético, floculados con polímero aniónico de alto peso molecular. Posteriormente, se evaluó el impacto de usar agua de mar tratada, reduciendo su contenido de calcio y magnesio, mediante una atmósfera rica en $\mathrm{CO}_{2}$ y utilizando $\mathrm{NaOH}$ como agente alcalinizante.

Los resultados muestran que el agua de mar genera un comportamiento eficiente al trabajar a un $\mathrm{pH}$ menor a 9. Sin embargo, a mayor alcalinidad la concentración de sólidos en el sedimento se reduce de manera considerable. Al analizar una solución salina con $\mathrm{NaCl}$ a la concentración que presenta el sodio en el agua de $\operatorname{mar}(0.5 \mathrm{M})$ se observa que la presencia de electrolitos no es un problema mayor, ya que el porcentaje de sólidos se preserva en todo el rango de $\mathrm{pH}$ estudiado.

Sin embargo, al analizar separadamente los principales iones divalentes que están presentes en agua de mar se encuentra que el magnesio, y en menor medida el calcio, son los responsables de disminuir la concentración de sólidos producto de la formación de complejos $\mathrm{MgOH}^{+} / \mathrm{CaOH}^{+}$y precipitados de $\mathrm{Mg}(\mathrm{OH})_{2}$ que bajan la eficiencia del floculante. Por este motivo, al utilizar el agua de mar con reducción de calcio y magnesio se logró disminuir sustancialmente la cantidad de agua contenida en el sedimento de las pulpas, ofreciendo una alternativa eficaz para operar a condiciones altamente alcalinas.

\section{AGRADECIMIENTOS}

Los autores agradecen el apoyo económico de CONICYT FONDECYT 11171036; CONICYT PIA ACM 170005; y a INNOVA CORFO CSIRO Chile 10CEII-9007. Pedro Robles agradece a la Pontificia Universidad Católica de Valparaíso por el apoyo proporcionado.

\section{REFERENCIAS}

Al-Anezi, K., N. Hilal, Effect of Carbon Dioxide in Seawater on Desalination: A Comprehensive Review, https://doi.org/10.1080/15422110600867365, Sep. Purif. Rev., 35, 223-247 (2006)

Antunes, E., F.A.P. Garcia y otros cuatro autores, Effect of Water Cationic Content on Flocculation, Flocs Resistance and Reflocculation Capacity of PCC Induced by Polyelectrolytes, Ind. Eng. Chem. Res., https://doi.org/10.1021/ie800326z, 47, 6006-6013 (2008)

Arias, D., M. Rivas y R. Guiñez, L.A. Cisternas, Modeling the Calcium and Magnesium Removal from Seawater by Immobilized Biomass of Ureolytic Bacteria Bacillus Subtilis through Response Surface Methodology and Artificial Neural Networks, doi: 10.5004/dwt.2018.22665, Desalin. Water Treat., 118, 294-303 (2018)

Atesok, G., P. Somasundaran y L.J. Morgan, Adsorption Properties of $\mathrm{Ca}^{2+}$ on Na-kaolinite and its Effect on Flocculation Using Polyacrylamides, https://doi.org/10.1016/0166-6622(88)80009-X, Colloids Surfaces A Physicochem. Eng. Asp. 32, 127-138 (1988)

Avadiar, L., Y.K. Leong y A. Fourie, Physicochemical Behaviors of Kaolin Slurries with and without Cations-Contributions of Alumina and Silica Sheets, https://doi.org/10.1016/j.colsurfa.2014.12.019, Colloids Surfaces A Physicochem. Eng. Asp., 468, 103-113 (2015)

Castro, S., Challenges in flotation of Cu-Mo Sulfide Ores in Sea Water I, in: Water in Mineral Processing. 29-40 (2012)

Castro, S., Use of Seawater and other saline water (salinity between 1-7\%) pre-treated with lime or other alkalinizing reagents, on mineral flotation process, wherer the pre-treatment removes the ions of seawater hardness without significantly altering its salinity, Chilean Patent, 52248 (2010)

Cisternas, L.A. y E.D. Gálvez, The Use of Seawater in Mining, https://doi.org/10.1080/08827508.2017.1389729, Miner. Process. Extr. Metall. Rev. 39, 18-33 (2018) 
Colic, M., G.V. Franks, M.L. Fisher y F.F. Lange, Effect of Counterion Size on Short Range Repulsive Forces at High lonic Strengths, doi: 10.1021/la960965p, Langmuir, 13, 3129-3135 (1997)

De Araujo, A.C., G.E.S. Valadao, E.M. Da Gama y C.A. Hernández, Consistencia, Fluidez y Viscosidad de Pastas Minerales de Relaves de Hierro, http://dx.doi.org/10.4067/S0718-07642006000200010, Inf. tecnol., 17 (2), $71-79$ (2006)

De Kretser, R., P.J. Scales, D.V. Boger, Improving Clay-Based Tailings Disposal: Case Study on Coal Tailings, https://doi.org/10.1002/aic.690430724, AIChE J., 43(7), 1894-1903 (1997)

Hernández, P.C., H.R. Galleguillos y otros tres autores, Effect of Seawater on the Solubility and Physicochemical Properties of Acidic Copper Sulfate Solutions, https://doi.org/10.1021/je300444r, J. Chem. Eng. Data, 57, 2430-2436 (2012)

Jeldres, R.I., M.P. Arancibia-Bravo y otros cuatro autores, The Impact of Seawater with Calcium and Magnesium Removal for the Flotation of Copper-Molybdenum Sulphide Ores, Miner. Eng, https://doi.org/10.1016/j.mineng.2017.02.003, 109, 10-13 (2017a)

Jeldres, R.I., L. Forbes y L.A. Cisternas, Effect of Seawater on Sulfide Ore Flotation: A Review, https://doi.org/10.1080/08827508.2016.1218871, Miner. Process. Extr. Metall. Rev. 37, 369-384 (2016)

Jeldres, R.I., E.C. Piceros y otros tres autores, Viscoelasticity and Yielding properties of Flocculated Kaolinite Sediments in Saline Water, https://doi.org/10.1016/j.colsurfa.2017.07.006, Colloids Surfaces and Eng. Asp., 529, 1009-1015 (2017b)

Jeldres, R.I., P.G. Toledo y otros tres autores, Impact of Seawater Salts on the Viscoelastic Behavior of Flocculated Mineral Suspensions, Colloids Surfaces A Physicochem. Eng. Asp., https://doi.org/10.1016/j.colsurfa.2014.08.003, 461, 295-302 (2014)

Ji, Y., Q. Lu, Q. Liu y H. Zeng, Effect of Solution Salinity on Settling of Mineral Tailings by Polymer Flocculants, https://doi.org/10.1016/j.colsurfa.2013.04.006, Colloids Surfaces A Physicochem. Eng. Asp., 430, $29-38$ (2013)

Lee, B.J., M.A. Schlautman, E. Toorman y M. Fettweis, Competition between Kaolinite Flocculation and Stabilization in Divalent Cation Solutions Dosed with Anionic Polyacrylamides, https://doi.org/10.1016/j.watres.2012.07.056, Water Res. 46, 5696-5706 (2012)

$\mathrm{Li}, \mathrm{W}$. , Y. Li y otros tres autores, The Influencing Mechanisms of Sodium Hexametaphosphate on Chalcopyrite Flotation in the Presence of $\mathrm{MgCl} 2$ and $\mathrm{CaCl}$, https://doi.org/10.3390/min8040150, Minerals, 8(4), 150 (2018)

Liu, D., M. Edraki y L. Berry, Investigating the Settling Behaviour of Saline Tailing Suspensions Using Kaolinite, Bentonite, and Illite Clay Minerals, https://doi.org/10.1016/j.powtec.2017.11.070, Powder Technol., 326, 228-236 (2018)

Maclver, M.R. y M. Pawlik, Measurement of Optical Backscattering Height Scans from Flocculated Mineral Sediments, https://doi.org/10.1016/j.colsurfa.2016.11.030, Colloids Surfaces A Physicochem. Eng. Asp., 514, 38-46 (2017)

Mpofu, P., J. Addai-Mensah y J. Ralston, Influence of Hydrolyzable Metal Ions on the Interfacial Chemistry, Particle Interactions, and Dewatering Behavior of Kaolinite Dispersions, https://doi.org/10.1016/S0021-9797(03)00113-9, J. Colloid Interface Sci., 261, 349-359 (2003)

Ordóñez, J.I., L. Moreno, E.D. Gálvez y L.A. Cisternas, Seawater Leaching of Caliche Mineral in Column Experiments, https://doi.org/10.1016/j.hydromet.2013.07.009, Hydrometallurgy, 139, 79-87 (2013)

Pan, L. y R.-H Yoon, Effec of Electrolytes on the Stability of Wetting Films: Implications on Seawater Flotation, doi: 10.1016/j.mineng.2018.03.011, Miner. Eng., 122, 1-9 (2018)

Peng, F.F. y P. Di, Effect of Multivalent Salts-Calcium and Aluminum on the Flocculation of Kaolin Suspension with Anionic Polyacrylamide, https://doi.org/10.1006/jcis.1994.1161, J. Colloid Interface Sci. 164, 229-237 (1994)

Quezada, G.R., R.I. Jeldres, P.D. Fawell y P.G. Toledo, Use of Molecular Dynamics to Study the Conformation of an Anionic Polyelectrolyte in Saline Medium and its Adsorption on a Quartz Surface, https://doi.org/10.1016/j.mineng.2018.09.025, Miner. Eng., 129, 102-105 (2018)

Ruan, Y., Z. Zhang y otros cuatro autores, Effects of Metal lons on the Flotation of Apatite, Dolomite and Quartz, doi: 10.3390/min8040141, Minerals, 8(4), 141 (2018)

Suyantara, G.P.W., T. Hirajima, H. Miki y K. Sasaki, Floatability of Molybdenite and Chalcopyrite in Artificial Seawater, https://doi.org/10.1016/j.mineng.2017.10.004, Miner. Eng., 115, 117-130 (2018)

Uribe, L., L. Gutierrez, J.S. Laskowski, S. Castro, Role of Calcium and Magnesium Cations in the Interactions Between Kaolinite and Chalcopyrite in Seawater, http://dx.doi.org/10.5277/ppmp170205, Physicochem. Prob. Mi., 53(2), 737-749 (2017)

Zbik, M.S., R.S.C. Smart y G.E. Morris, Kaolinite Flocculation Structure, https://doi.org/10.1016/j.jcis.2008.08.063, J. Colloid Interface Sci., 328, 73-80 (2008) 